Dermatologische Zeitschrift. 1933;67:I-IV

\title{
Contents, Vol. 67, 1933
}

\section{Inhaltsverzeichnis.}

Originalarbeiten.

Seite Beintema,K. und E. M. J. Jan sen, Pityriasis rubra pilaris generalisata unter dem Bilde einer figurierten Erythrodermie $\quad 222$

Berggreen, P., siehe Nagell, $\mathrm{H}$.

Bommer, S., Über Fluoreszenz der elastischen Fasern in der Haut . . 319

Emanuel, Sv., Blutung und Blutschädigung nacb Salvarsan 24

Hoffmann, Erich, Merkblatt über die Frühbehandlung der Syphilis

mit dem Ziel voller Ausheilung 161

Jan sen, E. M. J., siehe Beintema, K.

John, Ferdinand, Die Bedeutung der Gonokokken-Komplement-Bin-

dungsreaktion bei Frauen mit häufig wechselndem Geschlechtsverkehr 156

Keller, Philipp, Über Sklero-Poikilodermie 14

Nagell, H. und P. Berggreen, Über Kurzwellëntherapie bei Go-

norrhoe 151

Penning, C. P. J., siehe Zoon, J. J.

Richter,Wilhelm, Kapillarmikroskopische Studien über Frostschäden 147 Rotnes, Per L., Eine

Familien-Endemie von Erythema nodosum . . . 259 Schlammadinger, Josef, Phytogene

Hauterkrankungen, mit be-

sonderer Rücksicht auf die Wiesendermatitis324

Schmidt, Werner, Erfahrungen mit der Flavadinbehandlung der weib-

lichen Gonorrhoe 331

Schreiner, K., Zur Pathogenese der Salvarsan-Hautschäden und deren

Verhütung . 230

Stratmann,A., Über seltene Tumoren im Nagelbett (Neuromyoarterielle

Glomustumoren oder arterielle Angioneuromyome 129

Strempel, Rudolf, Über ausgedehnte multiple lupoide bzw. leproide

Granulome der Haut 1

Struycken, J., siehe Vonno, N. C. van.

siehe Zitzke, E.

siehe Teleky, L.

Teleky, L., Bemerkungen zur Arbeit von van Vonno und J. Struycken

(Bäckerekzem) 338

Vonno, N. C. van und Struycken, J., Untersuchungen über die Ent-

stehung des Bäckerekzems 43

- Weitere klinische und tierexperimentelle Untersuchungsergebnisse

über die Ursache der Berufsekzeme bei Bäckern. Erwiderung auf diese Arbeit von Dr. E. Zitzke in Bd. 65, H. 4/5 dieser Zeitschrift . 337

Woronoff, D. L., Über den Einfluß der Pyokokkeninfektion auf die 
Psoriasis vulgaris 253

Zitzke, Ern a, Weitere klinische und tierexperimentelle Untersuchungsergebnisse über die Ursache der Berufsekzeme bei Bäckern .... 209

- $\quad$ Schlußwort zu den Bemerkungen, von van Vonno und Struycken über

die Arbeit von Erna Zitzke betr. Entstehung des Bäckerekzems . . 338 Zoon, J. J. und C. P. J.

Penning, Zur Epidermolysis bullosa hereditaria

dystrophica 244

IV

Inhaltaverzeichnis.

Seit $\beta$

Sammelreferate.

Psoriasis, Lichenerkrankungen, Verhornungsanomalien, Pigmenta $\pi$ omalie $\pi$, Nagel- und Haarerkrankungen, Erkrankange $\pi$ der Talgdrüsen und

Schweißdrüsen. Sammelreferat vom Frühjahr 1932 bis Frühjahr

1933. Von Dr. H. 0. Loos -Innsbruck 83

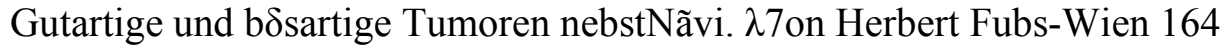

Strahlenheilkunde. Von Prof. Philipp Keller-Freiburg i. Br 265

Syphilis und andere Spirochätosen. Von R. Strempel und H. Kroó-

Bonn 340

Zusammenfassende Arbeiten diagnostischen und therapeutischen Inhalts.

Die Bedeutung und Wertung der Gonorrhoe-Komplementablenkung. Von

E. Kadisch-Berlin 119

Metastatische Hodenerkrankungen bei akaten Infektionskrankheiten, ihr

Vorkommen und ihre Erkennung. Von Dr. F. Callomon-Dessau . . 193

Über die diätetische Beeinflussung von Hautkrankheiten. Von Prof. Dr.

Paul Mulzer-Hamburg 282

Die Bedeutung des elektrischen Schneidens mit der Drahtschlinge fur die

Behandlung der Hautkrankheiten. Von Priv.-Doz. Dr. V. Wucher-

pfennig-Münster i. W 370

Qesellschaftsberichte.

Kölner Dermatologische Gesellschaft. Sitzung vom 24. Februar 1932 . . 201

Royal Society of Medicine. Section of Dermatology. Sitzung vom 15. Dezember $1932 \quad 202$

Sitzung vom 16. Februar 1933

Sitzung vom 16. März 1933

292

North of England, Dermatological Society. Sitzungen vom 19. Januar,

10. Februar und 28. April 1933 294

Société française de Dermatologie et de Syphiligraphie. Sitzungen vom

9. Juni und 7. Juli 1932

- $\quad$ Sitzung vom 10. November 1932

Buchbesprechungen 125, 204, 315, 391

Personalíe $\pi$ und Tagesnachrichten 128, 208, 318, 394

Joseph Jadassohn zum 70. Geburtstage

316

Bruno Bloch $\dagger$

126

Prof. Max Joseph und Prof. Ernst Kromayer $\dagger$

Prof. Viktor Mucha $\uparrow$ 